\title{
ENTIRE FUNCTIONS AND INTEGRAL TRANSFORMS
}

\section{BY LOUIS DE BRANGES}

Communicated by R. P. Boas, November 25, 1961

If $E(z)$ is an entire function which satisfies

$$
|E(\bar{z})|<|E(z)|
$$

for $y>0(z=x+i y)$, let $\mathfrak{F C}(E)$ be the corresponding Hilbert space of entire functions $F(z)$ such that

$$
\|F\|^{2}=\int|F(t) / E(t)|^{2} d t<\infty
$$

and

$$
|F(z)|^{2} \leqq\|F\|^{2}\left[|E(z)|^{2}-|E(\bar{z})|^{2}\right] /[2 \pi i(\bar{z}-z)]
$$

for all complex $z$. The space is introduced in [7], where it is characterized by three axioms. If $E(a, z)$ and $E(b, z)$ are entire functions which satisfy (1), then $\mathfrak{H C}(E(a))$ will be contained isometrically in $\mathfrak{H C}(E(b))$ if these functions satisfy the hypotheses of Theorem VII of [8]. Isometric inclusions of spaces of entire functions are a basic idea in [9] and [10]. A fundamental property of these inclusions has only now become available.

THeOREM I. If $E(a, z), E(b, z)$, and $E(c, z)$ are entire functions which satisfy (1) and have no real zeros, and if $\mathfrak{F C}(E(a))$ and $\mathfrak{F C}(E(b))$ are contained isometrically in $\mathfrak{H C}(E(c))$, then either $\mathfrak{H C}(E(a))$ contains $\mathfrak{H C}(E(b))$ or $\mathfrak{H C}(E(b))$ contains $\mathfrak{H}(E(a))$.

The formal proof depends on techniques of [2] and [3] for handling difference quotients. To make it precise, one must show that if $f(z)$ and $g(z)$ are entire functions of minimal exponential type such that

$$
|y f(z) g(z)| \leqq|f(z)|+|g(z)|
$$

for all complex $z$, then $f(z) g(z)$ vanishes identically. This is proved by a method of Carleman, for whose explanation we are indebted to M. Heins [16]. By Theorem III of [10], the theorem has applications for certain kinds of integral transforms.

THEOREM II. Let $u(x)$ and $v(x)$ be square integrable functions defined in $[0,1]$, such that

$$
\bar{u}(x) v(x)=\bar{v}(x) u(x)
$$

a.e., and which are essentially linearly independent when restricted to 
any subinterval of $[0,1]$. Let $T$ be the bounded linear transformation of $L^{2}(0,1)$ into itself defined by $T: g \rightarrow f$ if

$$
f(x)=\int_{x}^{1} g(t)[u(x) \bar{v}(t)-v(x) \bar{u}(t)] d t
$$

for almost all values of $x$. Let Tr be a closed subspace of $L^{2}(0,1)$ which is invariant under $T$ in the sense that $T g$ belongs to $\mathbb{N}$ whenever $g$ belongs to TT. Then, IT is characterized by a number a in $[0,1]$ and coincides with the set of functions which vanish a.e. for $x \geqq a$.

The same conclusion is available from the work of Kalisch [17] when $u(x)$ and $v(x)$ satisfy additional differentiability conditions. The point of Theorem II is that no such restrictions are necessary. Theorem II may be used to give a proof of uniqueness in the inverse Sturm-Liouville problem studied by Levinson [19].

THEOREM III. Let $\psi(x)$ be a uniformly continuous, increasing function of real $x$ such that

$$
\int\left(1+t^{2}\right)^{-1}|\psi(t)-\tau t|^{2} d t<\infty
$$

for some number $\tau>0$. If $0<a<\tau$, then there is a measure $\mu$ of finite total variation, supported in the points $t$ where $\psi(t) \equiv 0$ modulo $\pi$, such that $\int e^{i x t} d \mu(t)$ vanishes in $[-a, a]$ and does not vanish identically. Furthermore, the measure may be chosen of this special form: There is an entire function $S(z)$ of exponential type a which is real for real $z$ and has only real simple zeros, all at points $t$ where $\psi(t) \equiv 0$ modulo $\pi$, and

$$
\int\left(1+t^{2}\right)^{-1} \log ^{+}|S(t)| d t<\infty
$$

and

$$
\sum_{S(t)=0}\left|S^{\prime}(t)\right|^{-1}<\infty .
$$

The measure $\mu$ is supported in the zeros of $S(z)$ and has mass $S^{\prime}(t)^{-1}$ at each such zero $t$.

The formal part of the proof depends on the formula of [6] to obtain a measure, and on the convexity methods of [4] and [5] to obtain an entire function. To implement these procedures, we use a theorem of Beurling and Malliavin [20]: If $K(z)$ is an entire function of exponential type which satisfies (2), then for each $a>0$ there is a nonzero entire function $F(z)$ of exponential type $a$, bounded on the 
real axis, such that $K(z) F(z)$ is bounded on the real axis. Under the hypotheses of Theorem III, an entire function of minimal exponential type, which remains bounded on the set of points $t$ where $\psi(t) \equiv 0$ modulo $\pi$, is necessarily a constant. We should like to acknowledge our indebtedness to Chapter VIII of Levinson [18], which suggested the above theorem. The results of Levinson, Chapter IX, can be significantly bettered on using another theorem of Levinson, as it is formulated in [3]. The trick is to use Theorem XII of [9] to convert a result on nonvanishing Fourier transforms into an existence theorem for entire functions of minimal exponential type.

Theorem IV. Let $\left(a_{n}, b_{n}\right)$ be a sequence of disjoint intervals to the right of $x=1$ with lengths $b_{n}-a_{n}$ bounded away from zero and with

$$
\sum\left(b_{n}-a_{n}\right)^{2} a_{n}^{-1} b_{n}^{-1}=\infty \text {. }
$$

Then there exists an entire function of minimal exponential type which remains bounded on the real complement of $U\left(a_{n}, b_{n}\right)$ and is not a constant.

\section{REFERENCES}

1. R. P. Boas, Jr., Entire functions, Academic Press, New York, 1954.

2. L. de Branges, Local operators on Fourier transforms, Duke Math. J. 25 (1958), $143-154$.

3. - The a-local operator problem, Canad. J. Math. 11 (1959), 583-592.

4. - The Stone-Weierstrass theorem, Proc. Amer. Math. Soc. 10 (1959), 822-824.

5. - The Bernstein problem, Proc. Amer. Math. Soc. 10 (1959), 825-832.

6. - Some mean squares of entire functions, Proc. Amer. Math. Soc. 10 (1959), 833-839.

7. - Some Hilbert spaces of entire functions, Proc. Amer. Math. Soc. 10 (1959), 840-846.

8. - Some Hilbert spaces of entire functions, Trans. Amer. Math. Soc. 96 (1960), 259-295.

9. - Some Hilbert spaces of entire functions. II, Trans. Amer. Math. Soc. 99 (1961), 118-152.

10. - Some Hilbert spaces of entire functions. III, Trans. Amer. Math. Soc. 100 (1961), 73-115.

11. - Some Hilbert spaces of entire functions. IV, Trans. Amer. Math. Soc. (to appear).

12. - Some Hilbert spaces of entire functions. V, Trans. Amer. Math. Soc. (to appear).

13. - Homogeneous and periodic spaces of entire functions, Duke Math. J. (to appear).

14. - Symmetry in spaces of entire functions, Duke Math. J. (to appear).

15. - A comparison theorem for spaces of entire functions, Proc. Amer. Math. Soc. (to appear). 
16. M. Heins, On a notion of convexity connected with a method of Carleman, J. Analyse Math. 7 (1959), 53-77.

17. G. K. Kalisch, On similarity, reducing manifolds, and unitary equivalence of certain Volterra operators, Ann. of Math. 66 (1957), 481-494.

18. N. Levinson, Gap and density theorems, Amer. Math. Soc. Colloq. Publ. Vol. 26, Amer. Math. Soc., Providence, R. I., 1940.

19. — The inverse Sturm-Liouville problem, Nordisk Mat. Tidskr. B 49 (1949), 25-30.

20. P. Malliavin, Some applications of entire functions of exponential type to harmonic analysis, mimeographed notes from the conference on functional analysis at Stanford, 1961.

New York UnIversity 